\title{
Surveillance of Cystic Lesions of the Pancreas: Whom and How to Survey?
}

\author{
Stefano Andrianello ${ }^{a}$ Massimo Falconi $^{b}$ Roberto Salvia ${ }^{a}$ Stefano Crippa ${ }^{b}$ \\ Giovanni Marchegiani ${ }^{a}$ \\ a Pancreatic Surgery Unit of the Department of Surgery, Verona University Hospital, Verona, Italy; \\ bPancreatic Surgery Unit, Pancreas Translational and Clinical Research Centre, San Raffaele Scientific Institute, 'Vita-Salute' University, \\ Milan, Italy
}

\section{Keywords}

Pancreatic cystic neoplasms · Pancreatic cancer . Surveillance $\cdot$ Guidelines

\section{Summary}

The sudden prevalence increase of pancreatic cystic neoplasms (PCN) related to the use of cross-sectional imaging has raised several concerns. Even if there is a tangible risk of progression towards pancreatic cancer (PC), surgical resection cannot be offered to all patients due to the high risk of morbidity and mortality. Available guidelines are useful tools to identify patients at higher risk for harboring cancer thanks to their sensitivity. Because of their low specificity, however, such a risk is often overestimated. Recent evidence deriving from large observational series of surveilled patients suggests that the overall risk of $\mathrm{PC}$ is low. A large proportion of patients affected by PCN can be safely observed over time. Several follow-up schedules have been proposed in guidelines but none of them proved to be the most cost-effective. Moreover, it must still be demonstrated that any surveillance protocol can be associated with a reduction in PC-related mortality. By now, with most studies reporting a lifelong risk of malignancy, the only evidencebased recommendation regarding surveillance is that follow-up should never be discontinued as repeated observations are crucial for PC risk stratification.

(C) 2018 S. Karger GmbH, Freiburg

\section{Introduction}

Due to the extensive use of cross-sectional imaging, the prevalence of incidentally discovered pancreatic cystic lesions has dramatically risen, ranging from 1.2 to $36.7 \%$ in the general population [1-5] and with intraductal papillary mucinous neoplasms (IPMN) accounting for at least $80 \%$ of them [6]. As IPMN may progress to cancer following the adenoma-carcinoma sequence [7-9], these represent the main population as well as where to address efforts in order to prevent pancreatic cancer (PC). To avoid the indiscriminate use of high-risk surgical procedures, e.g. major pancreatic resections, guidelines have been drafted in the last two decades [10-14]. These guidelines, however, have a low level of evidence since they are based on expert opinion and retrospective series of pancreatic cystic neoplasms (PCN) that basically consider only surgically treated patients. This selection bias limits the knowledge regarding a vast portion of PCN individuals, i.e. those under surveillance. For this reason, guidelines have led to a clinical management characterized by high sensitivity, but low specificity, in predicting PC $[15,16]$. Both clinical and radiological predictors have been extensively correlated to malignant progression; however, their role concerning the population under surveillance is largely unknown.

Only recently, several large observational series [17-22] including both surgically treated and surveilled patients allowed to gain a wider point of view with promising evidence available to better define the population that could be safely kept under surveillance.

This review focuses on how to identify patients affected by PCN which could be safely sent to follow-up according to what is reported by both the guidelines and the new evidence coming from observational series.

Stefano Crippa and Giovanni Marchegiani share the senior authorship.

\section{KARGER}

() 2018 S. Karger GmbH, Freiburg

Fax +497614520714
Stefano Crippa, MD, PhD

Pancreatic Surgery Unit, Pancreas Translational and Clinical Research Centre

San Raffaele Scientific Institute, 'Vita-Salute' University

Via Olgettina Milano 60, 20132 Milan, Italy

crippal.stefano@ hsr.it 
Table 1. Safe surveillance of pancreatic cystic neoplasms (PCN) as reported by the major guidelines

\begin{tabular}{|c|c|c|}
\hline & Criteria for surveillance & Follow-up schedule \\
\hline $\begin{array}{l}\text { IAP Guidelines (2016) } \\
\quad[12]\end{array}$ & $\begin{array}{l}\text { - absence of WF } \\
\text { - absence of HRS } \\
\text { - not suspicious EUS findings }\end{array}$ & $\begin{array}{l}>30 \mathrm{~mm} \text { : MRI/CT scan every } 3-6 \text { months; consider surgery in young patients } \\
20-30 \mathrm{~mm} \text { : EUS in } 3-6 \text { months; then up to } 1 \text { year alternating MRI to EUS; consider surgery in } \\
\text { young patients } \\
10-20 \mathrm{~mm} \text { : CT/MRI every } 6 \text { months for } 1 \text { year, then every } 12 \text { months for } 2 \text { years, then every } \\
2 \text { years } \\
<10 \mathrm{~mm} \text { : CT/MRI in } 6 \text { months, then every } 2 \text { years }\end{array}$ \\
\hline $\begin{array}{l}\text { European Consensus } \\
\text { Statement on PCN } \\
\text { Guidelines (2012) } \\
\text { [26] }\end{array}$ & $\begin{array}{l}\text { all of the following: } \\
\text { - cyst size }<40 \mathrm{~mm} \\
\text { - } \mathrm{MPD}<6 \mathrm{~mm} \\
\text { - no mural nodules } \\
\text { - asymptomatic } \\
\text { - cyst growth rate }<2 \mathrm{~mm} / \text { year } \\
\text { - normal serum CA } 19-9\end{array}$ & $\begin{array}{l}\text { MRI or EUS } \\
\text { - year 1: every } 6 \text { months } \\
\text { - year 2-5: every } 12 \text { months } \\
\text { - year }>5 \text { : every } 6 \text { months } \\
\text { - in case of increase in size: every } 6 \text { months }\end{array}$ \\
\hline AGA Guidelines [13] & $\begin{array}{l}\text { - size }<30 \mathrm{~mm} \\
\text { - no solid component } \\
\text { - absence of concerning features } \\
\text { at EUS performed when indicated } \\
\text { by guidelines }\end{array}$ & $\begin{array}{l}\text { MRI } \\
\text { - after } 1 \text { year from diagnosis, then every } 2 \text { years } \\
\text { - surveillance should be discontinued after } 5 \text { years from diagnosis }\end{array}$ \\
\hline
\end{tabular}

$\mathrm{WF}=$ Worrisome features; HRS = high-risk stigmata; EUS = endoscopic ultrasound; MRI = magnetic resonance imaging; $\mathrm{CT}=$ computed tomography; $\mathrm{MPD}=$ main pancreatic duct.

\section{Management according to Existing Guidelines}

\section{The International Association of Pancreatology (IAP) Guidelines}

The IAP guidelines were published for the first time in 2006 after the Sendai consensus conference [10]. After being validated by several case series, they soon showed major limitations: despite providing high diagnostic sensitivity for PC, approximately $75 \%$ of the resections were performed for benign disease $[15,23]$. The IAP guidelines were subsequently updated in 2012 [11] and in 2016 [12]. The 2012 version provides a risk stratification identifying three different categories of PCN that have been maintained in the last update; i.e., i) those PCN presenting with the so-called 'highrisk stigmata' (HRS), namely enhancing mural nodules $\geq 5 \mathrm{~mm}$, a main pancreatic duct (MPD) measuring $\geq 10 \mathrm{~mm}$, and obstructive jaundice; ii) those presenting with 'worrisome features' (WF), namely episodes of acute pancreatitis, cyst size $\geq 30 \mathrm{~mm}$, enhancing mural nodules $<5 \mathrm{~mm}$, thickened/enhancing cysts walls, MPD measuring 5-9 mm, lymphadenopathy, abrupt change in MPD caliber with distal atrophy of the pancreatic parenchyma, increased serum carbohydrate antigen (CA) 19-9 level, and cyst growth rate $\geq 5 \mathrm{~mm} / 2$ years; and iii) those without WF or HRS. The guidelines suggest an operation for those patients fit for surgery presenting with HRS due to the high risk of harboring malignancy [15]. In case of WF, they suggest further evaluation to better stratify the risk of malignancy when performing endoscopic ultrasound (EUS). In case of cytology suspicious for malignancy, suspect involvement of the MPD, and a definite mural nodule $\geq 5 \mathrm{~mm}$, surgery is suggested. In case of absence of these features at EUS as well as absence of WF or HRS at initial diagnosis, follow-up is suggested. Surveillance is scheduled according to cyst size (table 1).
Due to the subsequent updates, the IAP guidelines have shifted towards a more conservative approach with the aim of reducing the rate of false-positive results for malignancy [24, 25].

\section{The European Consensus Statements on Cystic Tumors of the Pancreas}

Published in 2013 after an European expert consensus [26], these guidelines also are in line with a more conservative approach, identifying two categories of PCN: i) those with risk factors, namely PCN $\geq 40 \mathrm{~mm}$, symptoms, mural nodules, MPD size $>6$ $\mathrm{mm}$, cyst growth rate $>2 \mathrm{~mm} /$ year, and increased serum level of CA 19-9; and ii) those without. The former category should be treated with surgical resection, the latter with surveillance according to a provided follow-up schedule (table 1). For the first time, the European guidelines recommend increasing the surveillance with shorter follow-up intervals after the fifth year from diagnosis as they report a heightened risk of malignancy after 5 years due to the age of the lesion. Another European expert consensus has taken place in 2015, and the updated version of the European guidelines will be published soon.

\section{The American Gastroenterological Association (AGA) Guidelines}

The most debated guidelines are those published by the AGA in 2015 [13]. Unlike the others, these guidelines only refer to asymptomatic PCN with involvement of the secondary ducts of the pancreas. Even in this case, two different categories of risk are identified: i) high-risk features, namely cysts measuring $\geq 30 \mathrm{~mm}$, a dilated MPD, and a solid component; and ii) low risk features, namely those patients with cysts $<30 \mathrm{~mm}$ and without solid com- 
ponents. In the presence of at least two high-risk features, patients should undergo EUS with fine needle aspiration to better assess the risk for PC. In case of worrying results at EUS, patients should undergo surgical evaluation. Otherwise, patients can be surveilled with magnetic resonance imaging starting 1 year after the diagnosis and then every 2 years. For the first time, the AGA guidelines suggest follow-up discontinuation after 5 years from diagnosis (table 1), supposing that the small risk of malignant progression in stable cysts is likely to be outweighed by the costs of surveillance and the risk of surgery.

\section{New Perspectives from Observational Studies}

The recent years have been characterized by the attempt to overcome previously published guidelines as these were burdened with an important selection bias. As they were derived by merely surgical series, there was a serious risk of overestimating the incidence of cancer based on specific risk factors. The multicentric series reported by Crippa et al. [17] evaluated outcomes of nonresected presumed IPMN, showing a $12 \%$ overall incidence of PC as well as a $60.2 \% 5$-year disease-specific survival for patients with HRS compared to $96.2 \%$ for patients with WF according to the IAP guidelines. This study reinforces the concept that, in presence of WF, follow-up is absolutely appropriate, whereas, due to the reduced survival, surgical resection is recommended in surgically fit patients with HRS. The study published by the group of the Mayo Clinic also considered a large sample of both resected and surveilled PCN [25]. They found a low 5-year risk of PC (2\%) for patients without HRS or WF according to the IAP guidelines, whereas the risk increased progressively in the case of WF (4.1\%) or HRS (49.7\%), suggesting a tailored approach for surveillance strategies. The study from the Memorial Sloan Kettering Cancer Center presents data extracted from one of the largest databases but focuses on a small group of patients affected by PCN with more than 5 years of follow-up [21]. These PCN change over time in $44 \%$ of cases, presenting an overall rate of progression towards PC of $1 \%$. Even if the overall risk of progression is low, and even lower if compared to the risk of postoperative death associated with major pancreatic resection [27], the observed rate of cancer at 5 years in the group of stable and indolent PCN was 31.3 per 100,000 , i.e. sensibly higher if compared to the age-adjusted incidence rate of 7.04 per 100,000 . According to these results, cyst size stability at 5 years does not preclude the risk of future growth and progression to PC. For this reason, follow-up cannot be discontinued after 5 years. The study from the Harvard Medical School considered a quite large sample of both surveilled and resected PCN with one of the longest median follow-ups (more than 80 months) [18]. Invasive cancer was found in $4.4 \%$ of the overall population, with a standardized incidence ratio of 18.8 per 100,000 at 5 years. The authors also identified a $0.9 \% 5$-year risk of developing PC in the population of patients with $\mathrm{PCN} \leq 1.5$ $\mathrm{cm}$. The series published by the Seoul National University Hospital has confirmed that the majority of cysts under surveillance is indolent and does not require surgical resection [20]. The authors also underlined the role of cyst growth rate and its association with the development of WF and HRS. In keeping with this data, Crippa et al. [28] evaluated the long-term outcomes in a cohort of 144 patients followed up for a median of 84 months. Although changes during follow-up were observed in 69 patients (48\%), new onset of WF/HRS was observed in 26 patients (18\%). WF and HRS developed after a median follow-up of 71 and 77.5 months, respectively, from diagnosis. Of note, independent predictors of WF/HRS development were size at diagnosis $>15 \mathrm{~mm}$, increase in number of lesions, main pancreatic duct growth rate $\geq$ $0.2 \mathrm{~mm} /$ year, and cyst growth rate $>1 \mathrm{~mm} /$ year. Therefore, based on these reports, a size smaller than $15 \mathrm{~mm}$ and maybe cyst growth rate could be used as useful parameters to modulate surveillance strategies.

With regard to survival outcomes, the study from Karolinska University Hospital has reported a 5-year disease-specific survival rate for branch-duct IPMN (BD-IPMN) of approximately 100\%, even if radiological changes can be detected in the PCN of more than half of the patients during follow-up [19]. Again, the authors acknowledge the possibility of a safe surveillance for indolent BDIPMN without WF or HRS. The mid-term analysis of the NSPINAL study group showed how the 3-year risk of developing PC was $1.2 \%$ and the standardized incidence ratio was 16.6 per 100,000 in BD-IPMN [22]. A previous review and meta-analysis that included studies published before 2016 showed similar results [29]. Radiological changes were experienced by $28 \%$ of patients, and the overall incidence of malignancy was $3.7 \%$. Even in this case, the authors concluded that the risk of mortality due to PC and preventive surgical resection was similar, suggesting a non-operative management for BD-IPMN at low risk, as reported in the IAP guidelines. A more recent meta-analysis has meanwhile reported interesting results about IPMN with WF or HRS under surveillance in patients that are unfit for surgery [30]. The authors have stated that, in this particular population, IPMN-related mortality is low and considerably lower when compared to the risk of death for other causes. This suggests that, in patients with severe comorbidities, a conservative management even in case of high-risk PCN can be justified since their prognosis is not affected by the risk of developing PC.

As a matter of fact, after the AGA guidelines first proposed surveillance discontinuation after 5 years, several studies have attempted to identify a subpopulation of PCN in which follow-up could be potentially safely discontinued $[18,20,21]$. However, it has not yet been demonstrated that a prolonged follow-up could reduce PC-related mortality thanks to early diagnosis or prevention. However, no study had sufficient power to demonstrate that follow-up can be discontinued in selected cases. Until an adequate level of evidence is reached in favor of one or the other approach, surveillance of PCN without indication for surgery should never be ceased. Several clinical and radiological features may help in predicting the risk of malignant progression, and repeated observation should in time be individualized according to that risk. 


\section{Conclusion}

The available guidelines seem to be useful for identifying patients at risk for development of PC with a high level of sensitivity. Due to the small specificity, however, non-malignant PCN are often resected, exposing patients to a high risk of morbidity and mortality related to major pancreatic resection. Recent observational studies on large series of surveilled patients have reduced the significance of some risk factors, highlighting that a large portion of patients with PCN can be safely observed due to the low risk of progression towards PC. Although it is not yet possible to know whether a surveillance protocol is associated with a reduction in PC-related mortality, the discontinuation of follow-up still cannot be recommended because of the existing lifelong risk of developing malignancy.

\section{Disclosure Statement}

The authors did not provide a conflict of interest statement.

\section{References}

1 Laffan TA, Horton KM, Klein AP, et al: Prevalence of unsuspected pancreatic cysts on MDCT. AJR Am J Roentgenol 2008;191:802-807.

2 Zhang X-M, Mitchell DG, Dohke M, Holland GA, Parker L: Pancreatic cysts: depiction on single-shot fast spin-echo MR images. Radiology 2002;223:547-553.

$\checkmark 3$ Fernández-del Castillo C, Targarona J, Thayer SP, Rattner DW, Brugge WR, Warshaw AL: Incidental pancreatic cysts: clinicopathologic characteristics and comparison with symptomatic patients. Arch Surg 2003;138:424-427.

4 Lee KS, Sekhar A, Rofsky NM, Pedrosa I: Prevalence of incidental pancreatic cysts in the adult population on MR imaging. Am J Gastroenterol 2010;105:2079-2084.

5 Laurent L, Vullierme M-P, Rebours V, et al: Estimation of the prevalence of intraductal papillary mucinous neoplasm of the pancreas in the French population through patients waiting for liver transplantation. United Eur Gastroenterol J 2017;5:499-503.

6 Chang YR, Park JK, Jang J, Kwon W, Yoon JH, Kim S: Incidental pancreatic cystic neoplasms in an asymptomatic healthy population of 21,745 individuals. Medicine (Baltimore) 2016;95:e5535.

7 Lafemina J, Katabi N, Klimstra D, et al: Malignant progression in IPMN: a cohort analysis of patients initially selected for resection or observation. Ann Surg Oncol 2013;20:440-447.

8 Adsay V, Mino-Kenudson M, Furukawa T, et al: Pathologic evaluation and reporting of intraductal papillary mucinous neoplasms of the pancreas and other tumoral intraepithelial neoplasms of pancreatobiliary tract. Ann Surg 2016;263:162-177.

9 Sohn TA, Yeo CJ, Cameron JL, et al: Intraductal papillary mucinous neoplasms of the pancreas: an updated experience. Ann Surg 2004;239:788-789.

10 Tanaka M, Chari S, Adsay V, et al: International consensus guidelines for management of intraductal papillary mucinous neoplasms and mucinous cystic neoplasms of the pancreas. Pancreatology 2006;6:17-32.

11 Tanaka M, Fernández-del Castillo $\mathrm{C}$, Adsay V, et al: International consensus guidelines 2012 for the management of IPMN and MCN of the pancreas. Pancreatology 2012;12:183-197.
12 Tanaka M, Fernández-del Castillo C, Kamisawa T, et al: Revisions of international consensus Fukuoka guidelines for the management of IPMN of the pancreas. Pancreatology 2017;17:738-753.

13 Vege SS, Ziring B, Jain R, Moayyedi P; Clinical Guidelines Committee; American Gastroenterology Association: American gastroenterological association institute guideline on the diagnosis and management of asymptomatic neoplastic pancreatic cysts. Gastroenterology 2015;148:819-822.

14 Del Chiaro M, Verbeke C, Salvia R, et al: European experts consensus statement on cystic tumours of the pancreas. Dig Liver Dis 2013;45:703-711.

15 Goh BKP, Thng C-H, Tan DMY, et al: Evaluation of the Sendai and 2012 International Consensus Guidelines based on cross-sectional imaging findings performed for the initial triage of mucinous cystic lesions of the pancreas: a single institution experience with 114 surgically treated patient. Am J Surg 2014;208: 202-209.

16 Goh BKP, Lin Z, Tan DMY, et al: Evaluation of the Fukuoka Consensus Guidelines for intraductal papillary mucinous neoplasms of the pancreas: results from a systematic review of 1,382 surgically resected patients. Surgery 2015;158:1192-1202.

17 Crippa S, Bassi C, Salvia R, et al: Low progression of intraductal papillary mucinous neoplasms with worrisome features and high-risk stigmata undergoing nonoperative management: a mid-term follow-up analysis. Gut 2017;66:495-506.

18 Pergolini I, Sahora K, Ferrone CR, et al: Long-term risk of pancreatic malignancy in patients with branch duct intraductal papillary mucinous neoplasm in a referral center. Gastroenterology 2017;153:1284-1294.e1.

19 Del Chiaro M, Ateeb Z, Hansson MR, et al: Survival analysis and risk for progression of intraductal papillary mucinous neoplasia of the pancreas (IPMN) under surveillance: a single-institution experience. Ann Surg Oncol 2017;24:1120-1126.

20 Han Y, Lee H, Kang JS, et al: Progression of pancreatic branch duct intraductal papillary mucinous neoplasm associates with cyst size. Gastroenterology 2018;154: 576-584.

21 Lawrence SA, Attiyeh MA, Seier K, et al: Should patients with cystic lesions of the pancreas undergo longterm radiographic surveillance?: results of 3024 patients evaluated at a single institution. Ann Surg 2017; 266:536-544.
22 Ohno E, Hirooka Y, Kawashima H, et al: Natural history of pancreatic cystic lesions: a multicenter prospective observational study for evaluating the risk of pancreatic cancer. J Gastroenterol Hepatol 2018;33:320328.

23 Goh BKP, Tan DMY, Thng C-H, et al: Are the Sendai and Fukuoka consensus guidelines for cystic mucinous neoplasms of the pancreas useful in the initial triage of all suspected pancreatic cystic neoplasms? A singleinstitution experience with 317 surgically-treated patients. Ann Surg Oncol 2014;21:1919-1926.

24 Kaimakliotis P, Riff B, Pourmand K, et al: Sendai and Fukuoka consensus guidelines identify advanced neoplasia in patients with suspected mucinous cystic neoplasms of the pancreas. Clin Gastroenterol Hepatol 2015;13:1808-1815.

25 Mukewar S, de Pretis N, Aryal-Khanal A, et al: Fukuoka criteria accurately predict risk for adverse outcomes during follow-up of pancreatic cysts presumed to be intraductal papillary mucinous neoplasms. Gut 2017;66:1811-1817.

26 Del Chiaro M, Verbeke C, Salvia R, et al: European experts consensus statement on cystic tumours of the pancreas. Dig Liver Dis 2013;45:703-711.

27 Vollmer CM, Sanchez N, Gondek S, et al: A root-cause analysis of mortality following major pancreatectomy. J Gastrointest Surg 2012;16:83-89.

28 Crippa S, Pezzilli R, Bissolati M, et al: Active surveillance beyond 5 years is required for presumed branchduct intraductal papillary mucinous neoplasms undergoing non-operative management. Am J Gastroenterol 2017;112:1153-1161.

29 Crippa S, Capurso G, Cammà C, Delle Fave G, Fernández-del Castillo C, Falconi M: Risk of pancreatic malignancy and mortality in branch-duct IPMNs undergoing surveillance: a systematic review and meta-analysis. Dig Liver Dis 2016;48:473-479.

30 Vanella G, Crippa S, Archibugi L, Arcidiacono PG Delle Fave G, Falconi M, Capurso G: Meta-analysis of mortality in patients with high-risk intraductal papillary mucinous neoplasms under observation. Br J Surg 2018;105:328-338. 